\title{
Evaluation of the effectiveness of Gold STAMP intervention on pressure ulcer reductions
}

\author{
Yuchi Young *1, David Hornick², Yan $\mathrm{Wu}^{3}$, John Kalamaras ${ }^{3}$, Robert Pruzek ${ }^{3}$ \\ ${ }^{1}$ Department of Health Policy Management and Behavior, School of Public Health, University at Albany, Albany, NY, United \\ States \\ ${ }^{2}$ Homedical Associates, Albany, NY, United States \\ ${ }^{3}$ Department of Epidemiology \& Biostatistics, School of Public Health, University at Albany, Albany, NY, United States
}

Received: April 28, 2015

DOI: $10.5430 /$ jnep.v5n10p91
Accepted: June 15, 2015

Online Published: July 27, 2015

URL: http://dx.doi.org/10.5430/jnep.v5n10p91

\begin{abstract}
Introduction: This prospective cohort study is to evaluate the effectiveness of the Gold STAMP (Success Through Assessment, Management and Prevention) intervention on reducing pressure ulcer (PU) incidence rates among nursing home residents in New York State.

Methods: Nursing home residents $(n=2,480)$ who did not have PU at baseline were followed to calculate PU incidence. Gold STAMP educational intervention included care assessment, care practice, communication, and collaboration. Covariates included socio-demographics, selected health conditions, and functional status. Kaplan Meier survival analyses were conducted after propensity score matching (PSM) to assess the impact of the Gold STAMP intervention on PU incidence rate reduction.

Results: Prevalent conditions were urinary incontinence (83\%), bowel incontinence (73\%), and arthritis (37\%). The KaplanMeier survival analyses indicated that the case group had a higher percentage of residents with no PUs after 6 months of follow-up compared to the control group (94\% vs. 85\%; $p=.06$ ).

Conclusions: The 9\% PU incidence rate reduction is both clinically and financially meaningful. We suggest a longer study period to monitor the long-term effects of the Gold STAMP intervention in reducing PU incidence.
\end{abstract}

Key Words: Pressure, Ulcers, Incidence, Intervention, Propensity, Matching

\section{INTRODUCTION}

The prevalence and incidence of pressure ulcers (PU) are concerns for nursing homes. Much of the literature has concentrated on PU prevalence, cost of treatment, and increased mortality rates among patients living in nursing home settings. ${ }^{[1-4]}$ This literature tends to form a consensus that poor quality of life, multiple chronic conditions, and being immobile are consequences, and sometimes causes, of PU development and progression. ${ }^{[5]}$ It is thus difficult to disen- tangle the temporal relationship between these risk factors and PU incidence, and difficult to prescribe a set of effective interventions to reduce their occurrence of PUs.

PU prevalence and incidence varies depending on the study definition, severity measures and care setting. ${ }^{[2,3]}$ Incident cases of PUs are common in elderly nursing home patients who have never had PUs before. ${ }^{[3]}$ The incidence of PUs in nursing homes ranges from approximately $2.2 \%$ to $23.9 \%$, and from $0 \%$ to $17 \%$ for home care services. ${ }^{[3]}$ Unfortu-

\footnotetext{
*Correspondence: Yuchi Young; Email: yyoung@albany.edu; Address: Department of Health Policy Management and Behavior, School of Public Health, University at Albany, Albany, NY, United States.
} 
nately, recurrence is likely $70 \%$ of seniors 65 years of age and older who had a prior PU will develop a new PU. ${ }^{[6]}$ The annual cost of treating PUs is estimated to be between 1.3 and 3.5 billion US dollars ${ }^{[7]}$ the average hospital treatment cost for stage IV PUs is approximately $\$ 125,000 .{ }^{[8]}$ For obvious clinical and financial reasons, prevention is preferable.

The predisposing conditions that contribute to PU development are complex and treatment modalities vary. Studies have attempted to use educational interventions to reduce PU rates. Morente, Morales-Asencio, \& Veredas ${ }^{[9]}$ found that a self-learning, e-learning tool produced significantly better learning acquisition results than traditional in-class sessions for PU prevention. Olsho et al. ${ }^{[10]}$ examined the effect of On-Time clinical decision support to produce timely risk information, improve communication, strengthen care plans and preventive practices, and reduce PU incidence. This study found that joint implementation of 4 core On-Time components was associated with a significant reduction in PU incidence. In addition to educational and technological interventions, leadership and communication training is essential to reduce PU incidence. Sharkey et al. ${ }^{[11]}$ found that high implementation of On-Time quality improvement for PU prevention depended on a high level of involvement from the administrator, director of nursing, and the care team. Frumenti and Kurtz ${ }^{[12]}$ also found that a leadership intervention to focus on working with three different aspects of PU management reduced the PU prevalence rate from $10.59 \%$ to $7.76 \%$ for the intervention group.

This clinician education training program - Gold Success Through Assessment, Management and Prevention (Gold STAMP) is a PU reduction program developed by the New York State (NYS) Department of Health (DOH). It is a statewide initiative that brought together healthcare professionals from each nursing home to lectures and seminars to discuss best practices to prevent PUs. This evaluation quantifies the impact of Gold STAMP on the reduction of PU incidence among residents of nursing homes participating in the collaboratives vs. residents of non-participating nursing homes.

\section{Methods}

\subsection{Study design and study site}

This is a cohort study from September 1, 2011 to September 31, 2012. All nursing homes under study were part of four collaboratives in New York State (Buffalo, Rochester, Binghamton, and Kingston). Spearheaded by the NYSDOH and the Healthcare Association of New York State (HANYS), four collaboratives consisting of at least one hospital, one nursing home, and one home health care agency were formed to campaign for PU reduction. Due to data availability, this evaluation includes only patients of nursing homes.

\subsection{Study population}

Residents of nursing homes who did not have PUs at baseline and who were alive during the study period were selected for the study. PU assessments were based on Minimum Data Set (MDS) data.

\subsection{Case group $(n=644)$}

All residents of the seven nursing homes from the four collaboratives constitute the case group. To provide a control group, other nursing home facilities in the catchment areas (but not in the case group) were first matched with case group nursing homes based on the following criteria: 1) location in the DOH-defined catchment areas (Buffalo, Rochester, Binghamton, and Kingston), 2) PU prevalence, and 3) number of beds. Based on these criteria there were 43 matching nursing homes available for the control group, we randomly selected 15 nursing homes to serve as controls $(n=1,836)$. The case and control nursing home matching ratio was 1:2.

\subsection{Data source}

The data acquisition and use agreements were in compliance with the NYSDOH and University at Albany Institutional Review Board (IRB). In addition to the general IRB approvals from NYSDOH and the University at Albany, the evaluation team completed separate applications for a Data Use Agreement for secondary data sets from the MDS. MDS data from January 1, 2007 - December 31, 2012 were obtained. The data set comprised baseline measures of sociodemographics, health characteristics, functional limitations and PU incidence.

\subsection{Intervention}

The Gold STAMP educational intervention included a training component which covered the following topics: care assessment (e.g., PU risk and skin assessment, documentations), care practice (e.g., repositioning, support surfaces), and communication (e.g., care coordination, discharge plan, transfer of care, use of standardized PU communication tool between acute and long-term care settings). The target audience of the educational training program were direct care staff (e.g., RNs, PAs or NPs, nutritionists) from the case group. Four in-class training sessions lead by two PU clinical specialists were conducted in Albany, NY and subsequent phone conferences, and email consultations and support were available to attendees. Some study sites took the advantages of the consultation opportunity. Also, on-site visit by the PU clinical specialists were available upon request. Pressure ulcer monthly reports were submitted to the Educational Team for monitoring purpose. The intervening variable was 
whether or not the nursing home received the Gold STAMP educational training (yes vs. no).

\subsection{Outcome variable}

The outcome variable was the incidence rate of PUs. Incidents or new cases refer to patients who did not have PU at baseline but subsequently developed PUs including stages $1,2,3$, or 4 during the study period. The incidence rate was calculated using total the number of new cases divided by the total number of nursing home residents at risk. PU incidence information was not affected by the assessment forms changes from MDS2 to MDS3 in 2010 because we were only interested in the presence (yes $=1$ ) or absence (no $=0$ ) of PUs, not the severity.

\subsection{Control variables}

The covariates were sociodemographics, selected health conditions and functional disabilities using activities of daily living (ADL) measures. The sociodemographics were age, gender, race (White $v s$. non-White) and marital status (currently married: yes $v s$. no). The health conditions included heart failure, pneumonia, diabetes mellitus, arthritis, hip fracture, depression, urinary incontinence, bowel incontinence (yes vs. no), weight loss (malnutrition), and body mass in$\operatorname{dex}(\mathrm{BMI})$ as a categorical variable with three levels $(<17$, $17-24,>24)$. Chronic health condition was a dichotomous variable (active diagnosis: yes vs. no). Physical function was a summary variable measured by ADL tasks (bed mobility, eating, bathing, dressing, transferring, personal hygiene, and toileting); the five response levels included independent, supervision needed, limited assistance, extensive assistance, and total dependence. The values assigned each response level were 0-4, with higher scores indicating greater functional limitation.

\subsection{Data analysis}

Summary statistics were generated to describe both clinical and non-clinical characteristics of these 2,480 residents. To minimize selection bias between the intervention and reference groups, we conducted propensity score matching (PSM) to quantify the impact of the intervention after adjusting for covariates that predict intervention vs. control group membership. To estimate the propensity scores, we first developed a logistic regression model with the dependent variable being selection for intervention (Yes or No), and the independent variables age, gender, race, urinary tract infection, ADL disability, collaborative effort and their interaction terms, finalized by backward selection (data not shown). Then we matched the propensity scores estimated from the logistic regression to provide the conditional probability of selection for the intervention and control groups using a 1:1 Greedy matching algorithm. The sample size was 2,480 before propensity score matching (PSM) (add PSM citation) and 1,016 after PSM.

Since attrition from the study is possible by right censoring due to death, discharge or transfer prior to developing any PUs, a Kaplan-Meier survival analysis was performed to estimate the time to the event, in this case PU development. The survival curves estimate the trajectory of PU development for the two groups, allowing the evaluators to assess the benefit of the intervention, even if only moderate benefit is apparent in the short term. Statistical significance was established at a $p$-value less than .05. Analyses were performed in SAS 9.2 (SAS Institute, Cary NC).

\section{RESULTS}

A summary of selected socio-demographic and health characteristics of the study population is presented in Table 1. The average age of the study sample was 83 years and 54\% were in the oldest age group (85 years or more). Participants were predominately female $(71 \%)$ and White $(89 \%)$, and nearly 1 in 5 were currently married (19\%). Comorbidities included urinary incontinence (83\%) and bowel incontinence $(73 \%)$, followed by depression (54\%), arthritis (37\%), diabetes mellitus (33\%) and heart failure (28\%). A high level of functional disability was observed among residents (mean $\mathrm{ADL}=2.9$ on a $0-4$ scale), indicating that residents required extensive assistance for ADL tasks.

While the difference between the intervention and control groups did not reach statistical significance, it is fair to conclude that the Gold STAMP intervention confers added benefit. The intervention group experienced a lower incidence rate $(9 \%)$ compared to the control group $(11 \%)$ at baseline prior to the intervention $(p=.09)$. Following PSM, the Kaplan-Meier curve results indicated that the intervention group had a lower PU incidence rate after 6 months post intervention follow-up ( $6 \%$ vs. $15 \% ; p=.06)$.

Figure 1 demonstrates how propensity scores were used to reduce selection bias by equating groups based on covariates. Figure 1a shows frequency distributions of covariate-based propensity scores for before and Figure 1b after PSM. As is evident in Figure 1a, the propensity score distribution varied between the two groups, meaning the case and control groups were quite different before matching. The postmatching graph (see Figure 1b) shows that the propensity score distributions overlapped for both the intervention and reference groups after taking into account the covariate effects (i.e., age, gender, race, ADL, urinary tract infection (UTI), collaborative membership and interaction terms for the intervention). 
Table 1. Selected socio-demographic and health-related characteristics of the study population before PSM

\begin{tabular}{|c|c|c|c|c|}
\hline & Total $(N=2480)$ & Intervention $(\mathrm{N}=644)$ & Comparison $(\mathrm{N}=1836)$ & $\boldsymbol{P}$ \\
\hline \multicolumn{5}{|l|}{ Sociodemographics } \\
\hline \multicolumn{5}{|l|}{ Age } \\
\hline$<65$ & $171(7 \%)$ & $51(8 \%)$ & $120(7 \%)$ & \\
\hline $65-74$ & $226(9 \%)$ & $56(9 \%)$ & $170(9 \%)$ & \\
\hline $75-84$ & $740(30 \%)$ & 198 (31\%) & $542(30 \%)$ & \\
\hline $85+$ & $1,343(54 \%)$ & 399 (53\%) & $1,004(55 \%)$ & \\
\hline Mean (SD) & $83.19(11.08)$ & $82.80(11.30)$ & $83.33(11.01)$ & .29 \\
\hline Female & 1,761 (71\%) & 499 (77\%) & 1,262 (69\%) & \\
\hline White & 2,189 (89\%) & 588 (91\%) & $1,601(87 \%)$ & \\
\hline Currently married & 475 (19\%) & $105(16 \%)$ & $370(20 \%)$ & .03 \\
\hline \multicolumn{5}{|l|}{ Health characteristics } \\
\hline Heart failure & $692(28 \%)$ & $171(27 \%)$ & $521(28 \%)$ & .37 \\
\hline Pneumonia & $275(11 \%)$ & 57 (9\%) & $218(12 \%)$ & .04 \\
\hline Diabetes mellitus & 829 (33\%) & 208 (32\%) & $621(34 \%)$ & .48 \\
\hline Arthritis & 906 (37\%) & 229 (36\%) & 677 (37\%) & .49 \\
\hline Hip fracture & $115(5 \%)$ & $30(5 \%)$ & $85(5 \%)$ & .97 \\
\hline Depression & 1,399 (54\%) & $348(54 \%)$ & 991 (54\%) & .94 \\
\hline Urinary incontinence & 2,060 (83\%) & 559 (87\%) & $1,501(82 \%)$ & .006 \\
\hline Bowel incontinence & 1,809 (73\%) & $473(74 \%)$ & 1,336 (73\%) & .54 \\
\hline Weight loss & $441(18 \%)$ & $101(16 \%)$ & $340(19 \%)$ & .11 \\
\hline BMI & $28(7.28)$ & $27.55(6.6)$ & $28.15(7.5)$ & .07 \\
\hline Physical Function (ADL) & $2.88(1.03)$ & $2.75(1.1)$ & $2.92(1.0)$ & .003 \\
\hline Pressure ulcer incidence rates (Baseline)* & $262(11 \%)$ & 57 (9\%) & 205 (11\%) & .09 \\
\hline
\end{tabular}

*Pressure Ulcer incidence rate was calculated using total number of new cases divided by the total number of nursing home residents at risk.

Results of the Kaplan-Meier curves for the effectiveness of the Gold STAMP intervention are presented in Figure 2. The results show that there is a noticeable separation of PU rates between the case and control groups. Immediately after the intervention was completed, $3 \%$ of the residents in the intervention group had a PU compared to $5 \%$ of the residents in the reference group. The gap between the two curves contin- ued to widen at 6 months of follow-up: incidence rates were $9 \%$ vs. $11 \%$ for case and control groups, respectively. By 9 months of follow-up, the difference in PU incidence between the two groups was more noticeable at $6 \%$ for case $v s .15 \%$ for control groups. The $9 \%$ difference $(P=.06)$ between the two groups is clinically meaningful and has significant policy and financial implications for nursing home residents.

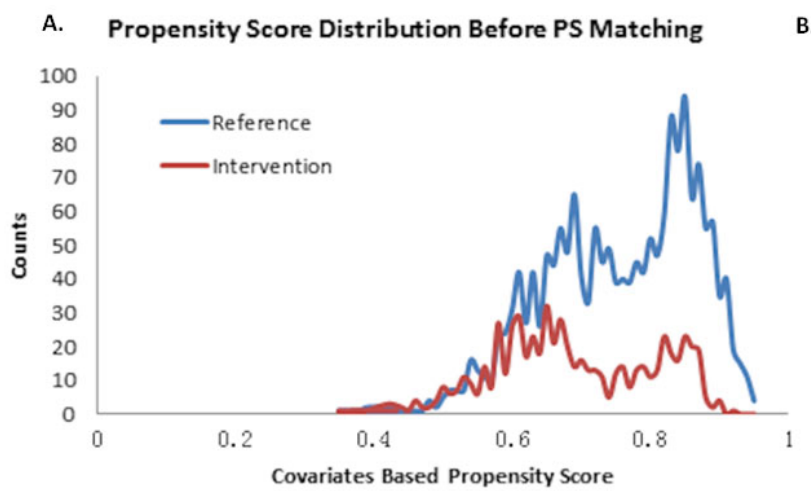

B.

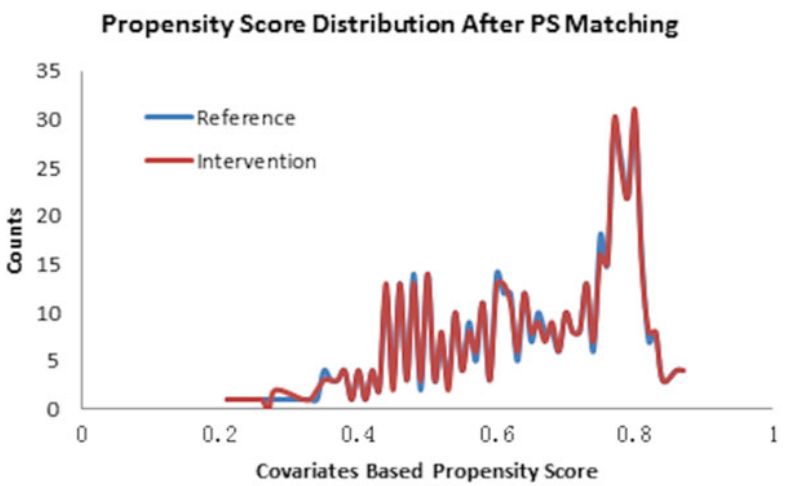

Figure 1. Propensity score matching (PSM) 

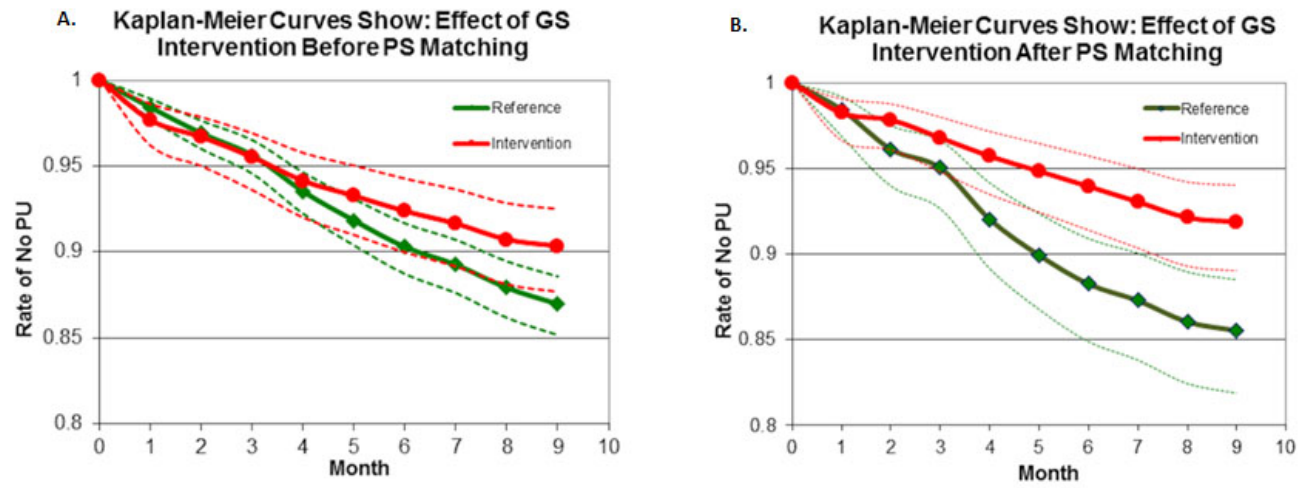

Figure 2. Kaplan-Meier results on the effectiveness of the intervention

Table 2. Estimates of logistic regression analysis of covariates and intervention

\begin{tabular}{lll}
\hline Parameter & Wald Chi-Square & $\boldsymbol{P}$ \\
\hline Age & .06 & .8 \\
Gender & 10.01 & .002 \\
Race & 8.47 & .004 \\
Functional disability (ADL) & 6.81 & .009 \\
Urinary tract infection (UTI) & 15.00 & $<.001$ \\
Collaborative (Rochester) & 31.78 & $<.001$ \\
Age $\times$ race & 5.08 & .02 \\
Gender $\times$ race & 4.03 & .04 \\
Age $\times$ collaborative & 12.21 & .007 \\
ADL $\times$ collaborative & 24.71 & $<.001$ \\
Race $\times$ collaborative & 63.41 & $<.001$ \\
Gender $\times$ collaborative & 15.31 & .002 \\
UTI $\times$ collaborative & 30.36 & $<.001$ \\
\hline
\end{tabular}

\section{Discussion}

Nursing home residents in NYS experienced a decline in independence and an increase in chronic conditions during our study period (data not shown), making the achievement of any reduction in PU incidence fairly remarkable. A slow but steadily declining trend in PU incidence in nursing homes was evident in NYS as well as in both the case and control groups. This could potentially be due to ongoing policies, programs, and care practices that are already addressing risk factors for PUs but which are unrelated to the Gold STAMP project, and thus not measured by our study. Our analytical methods took this possibility into account by using PSM to adjust for these secular trends among both case and control groups.

Our results are consistent with those of other studies related to educational interventions to reduce PUs. Although study design, intervention strategy employed, and outcomes of interest varied, positive findings have been documented. For Published by Sciedu Press example, in a similar study by Baier et al. ${ }^{[13]}$ which was conducted among nursing homes using a structured collaborative quality improvement approach, 6 of 8 prevention process measures improved significantly. In study by Abel, et al. ${ }^{[14]}$ a tool kit of materials was provided for nursing homes to implement the process of care system changes; education and training for nursing home staff was also provided. The process of care and patient outcome measures improved markedly for the nursing homes that implemented the prevention program. Ham, Schoonhoven, Schuurmans, Veugelers, \& Leenen ${ }^{[15]}$ found that providing PU education improved accurate identification from $87.7 \%$ to $95.6 \%$ and classification skills from $68.5 \%$ to $79.8 \%$. In another study, it was observed that adherence to long term care practices, such as standardized risk assessment and regular positioning, was as low as 50\%. ${ }^{[16]}$ Overall, interventions with an educational component helped clinical care givers recognize risk factors for the development of PUs. Better assessment coupled with improved management and tighter communication across clinical settings yields improvements in PU prevention.

In addition to many preventive educational, patient safety, and treatment programs to reduce PUs, the Centers for Medicare and Medicaid Services (CMS) implemented a new payment rule in 2008 that included PUs stages 3 and 4 in the "never events" category. Thus, hospitals are no longer reimbursed for treating nosocomial PUs in Stage III and IV. ${ }^{[17,18]}$ Other health policy initiatives that support PU prevention include the development of $\mathrm{AHRQ}^{[19]}$ and $\mathrm{CMS}^{[20]}$ guidelines for prevention and management of PUs. Now that PUs are considered an indicator of poor quality of care, demand for evidence-based prevention programs will grow and additional research should seek to validate our findings in other clinical settings and in home health care.

\section{Limitations of the study}

Several limitations may affect the generalizability of our findings to other nursing homes. Because all nursing home par- 
ticipants were voluntary, they may have been highly vested in achieving positive outcomes. Thus, the relatively narrow margin between the trajectories of our case and control groups might be widened if the intervention had included nursing homes which were not as far along in their PU reduction efforts. Data bias could be due to the assessment competencies of clinical staff, and data entry errors could influence the PU incidence and prevalence rates. A low percentage of residents with missing values in the covariates was removed from the study based on the missing completely at random (MCAR) assumption which is usually not true in observational data. Future studies can use multiple imputation methodology to deal with the missing data on a more relaxed assumption missing at random (MAR). The time horizon for follow-up is relatively short, and continuing follow-up on long-term outcomes is necessary to see whether these patterns persist. Finally, with respect to fidelity of implementation, the degree to which each collaborative maintains the observed success remains to be seen. Carefully documenting Gold STAMP training processes and the transfer of knowledge and skills to nurses or nursing assistants is essential for large scale program replication. Better integration of the Gold STAMP guidelines and care practices throughout nursing homes and home health care agencies may lead to further reductions in PU incidence. An evaluation of implementation fidelity is needed to ascertain the degree to which the knowledge and skills acquired from the training were implemented among nurses and nursing assistants who provide direct care for residents on a daily basis.

\section{Conclusion}

This evaluation suggests that the Gold STAMP intervention is effective in reducing PU incidence rates over a nine-month period among nursing home residents. This evaluation sheds light on the impact of Gold STAMP interventions on PU reduction. The time horizon for follow-up is relatively short so future studies should increase follow-up time. This may lead to further reduction in PU incidence. In addition, the cost-effectiveness study on Gold STAMP educational intervention has significant implications for policy makers and nursing home stakeholders.

\section{ACKNOWLEDGEMENTS}

The research was funded by the Medicaid Redesign Team and the New York State Department of Health (NYSDOH). Our appreciation is extended to the nursing homes and home health care agencies in Buffalo, Rochester, Binghamton, and the Kingston collaboratives. As active participants in the Gold STAMP training and service as champions for pressure ulcer reduction, they were invaluable to the project.

Many NYS DOH professionals were instrumental to this project's success. We are grateful for the leadership of Jacqueline O. Pappalardi of the NYSDOH, who provided our team with consistent guidance and support. We also acknowledge the intervention training efforts lead by Cheryl Reeves of School of Public Health Training Center at University at Albany. We specifically extend our gratitude to the following individuals for data access and management support: Mr. John Huffaker, Coordinator of MDS/OASIS/ASPEN Automation/DUA; Matthew Leary, Director and Gregory Schoonmaker, Program Research Specialist at NYSDOH BHCRIS; John R. Piddock, Director, Data Access Unit, Bureau of Biometrics and Health Statistics. This manuscript also benefited from the thoughtful editing of Allison Krug, MPH.

\section{CONFlicts OF INTEREST Disclosure}

The author declares that there is no conflict of interest statement.

\section{REFERENCES}

[1] Allman RM. Pressure ulcer prevalence, incidence, risk factors, and impact. Clin Geriatr Med. 1997 Aug; 13(3): 421-36. PMid:9227937

[2] Ayello EA, Sibbald RG. Geriatric Nursing Resources for Care of Older Adults: Pressure Ulcers \& Skin Tears. 2012. Available from: http://consultgerirn.org/topics/pressure_ulce rs_and_skin_tears/want_to_know_more

[3] Cuddigan J, Ayello EA, Sussman C, et al. Pressure ulcers in America: prevalence, incidence, and implications for the future. An executive summary of the National Pressure Ulcer Advisory Panel monograph. Advances in Skin \& Wound Care. 2001; 14(4): 208-215.

[4] Spoelhof GD, Ide K. Pressure ulcers in nursing home patients. Am Fam Physician. 1993 Apr; 47(5): 1207-15. PMid:8465716
[5] Horn SD, Sharkey SS, Hudak S, et al. Pressure ulcer prevention in long-term-care facilities: a pilot study implementing standardized nurse aide documentation and feedback reports. Adv Skin Wound Care. 2010 Mar; 23(3): 120-31. PMid:20177165 http: //dx.doi.org/10.1097/01.ASW.0000363516.47512.67

[6] Whittington KT, Briones R. National Prevalence and Incidence Study: 6-year sequential acute care data. Adv Skin Wound Care. 2004 Dec; 17(9): 490-4. PMid:15632743 http://dx.doi.org/10.1097/0 0129334-200411000-00016

[7] Courtney BA, Ruppman JB, Cooper HM. Save our skin: initiative cuts pressure ulcer incidence in half. Nurs Manage. 2006 Apr; 37(4): 36, 38, 40. PMid:16603946 http://dx.doi.org/10.1097/000 06247-200604000-00010

[8] Brem H, Maggi J, Nierman D, et al. High cost of stage IV pres- 
sure ulcers. Am J Surg. 2010 Oct; 200(4): 473-7. PMid:20887840 http://dx.doi.org/10.1016/j.amjsurg. 2009.12.021

[9] Morente L, Morales-Asencio JM, Veredas FJ. Effectiveness of an e-learning tool for education on pressure ulcer evaluation. J Clin Nurs. 2014 Jul; 23(13-14): 2043-52. PMid:24354930 http: //dx.doi.org/10.1111/jocn. 12450

[10] Olsho LEW, Spector WD, Williams CS, et al. Evaluation of AHRQ's on-time pressure ulcer prevention program: a facilitator-assisted clinical decision support intervention for nursing homes. Med Care. 2014 Mar; 52(3): 258-66. PMid:24374408 http://dx.doi.org/10.10 97/MLR. 0000000000000080

[11] Sharkey S, Hudak S, Horn SD, et al. Exploratory study of nursing home factors associated with successful implementation of clinical decision support tools for pressure ulcer prevention. Adv Skin Wound Care. 2013 Feb; 26(2): 83-92. PMid:23337649 http: //dx.doi.org/10.1097/01.ASW.0000426718.59326.bb

[12] Frumenti JM, Kurtz A. Addressing hospital-acquired pressure ulcers: patient care managers enhancing outcomes at the point of service. J Nurs Adm. 2014 Jan; 44(1): 30-6. PMid:24316616 http://dx.doi.org/10.1097/NNA.0000000000000018

[13] Baier RR, Gifford DR, Lyder CH, et al. Quality improvement for pressure ulcer care in the nursing home setting: the Northeast Pressure Ulcer Project. J Am Med Dir Assoc. 2003 Dec; 4(6): 291-301. http://dx.doi.org/10.1016/S1525-8610(04)70387-4

[14] Abel RL, Warren K, Bean G, et al. Quality improvement in nursing homes in Texas: results from a pressure ulcer prevention project. J Am Med Dir Assoc. 2005 Jun; 6(3): 181-8. PMid:15894247 http://dx.doi.org/10.1016/j.jamda.2005.03.011
[15] Ham WHW, Schoonhoven L, Schuurmans MJ, et al. Pressure ulcer education improves interrater reliability, identification, and classification skills by emergency nurses and physicians. J Emerg Nurs. 2015 Jan; 41(1): 43-51. PMid:24862184 http://dx .doi .org/10. $1016 / j \cdot j$ jen.2014.03.005

[16] Saliba D, Rubenstein LV, Simon B, et al. Adherence to pressure ulcer prevention guidelines: implications for nursing home quality. J Am Geriatr Soc. 2003 Jan; 51(1): 56-62. PMid:12534846 http://dx.doi.org/10.1034/j.1601-5215.2002.51010.x

[17] Delmore B, Lebovits S, Baldock P, et al. Pressure ulcer prevention program: a journey. J Wound Ostomy Continence Nurs. 2011 Oct; 38(5): 505-13. PMid:21860330 http://dx.doi.org/10.1097/W ON. Ob013e31822ad2ab

[18] Niederhauser A, VanDeusen LC, Parker V, et al. Comprehensive programs for preventing pressure ulcers: a review of the literature. Adv Skin Wound Care. 2012 Apr; 25(4): 167-88. PMid:22441049 http: //dx.doi.org/10.1097/01.ASW.0000413598.97566.d7

[19] Jarrett NM, Holt S, LaBresh KA. Evidence-Based Guidelines for Selected, Candidate, and Previously Considered Hospital-Acquired Conditions. 2014. Available from: http://sh.st/st/787f28ed3 e745c14417e4aec27303038/www.cms.gov/Medicare/Medic are-Fee-for-Service-Payment/HospitalAcqCond/Downl oads/Evidence-Based-Guidelines.pdf

[20] National Guideline Clearinghouse I Guideline for prevention and management of pressure ulcers. 2015. Available from: http://www . guideline. gov/content . aspx?id=23868 